\title{
Estimation of dynamic characteristics of a spring-mass-beam system
}

\author{
Ding Zhou ${ }^{\mathrm{a}, \mathrm{b}, *}$ and Tianjian $\mathrm{Ji}^{\mathrm{b}}$ \\ ${ }^{a}$ College of Civil Engineering, Nanjing University of Technology, Nanjing 210009, P.R. China \\ ${ }^{\mathrm{b}}$ School of Mechanical, Aerospace and Civil Engineering, The University of Manchester, Manchester, UK
}

\begin{abstract}
This paper presents an approximate solution for the analysis of the dynamic characteristics of a spring-mass-beam system. The spring-mass can be distributed or concentrated on a beam, which can represent a crowd or an individual on a beam. The analysis is based on the fact that a spring-mass-beam system can be modeled approximately as a series of two degree-of-freedom (TDOF) systems and the frequency coupling occurs mainly at the first TDOF system. The Galerkin method is used to derive the frequency equation of the TDOF system. Static beam functions of a beam with distributed and concentrated spring-masses are developed for the solutions, in which the effect of the magnitude and position of the mass of the spring-mass on the beam is considered. Using a set of simple formulae, the first pair of coupled frequencies and the corresponding mode can be obtained. The mass and stiffness factors in the TDOF system are tabled for engineering applications. For verification and use of the proposed method, a case of human-structure interaction is analysed using the proposed method and FE method. Parametric studies show that using the proposed functions, not only the first pair of natural frequencies but also the mode and internal forces of the coupled system can be obtained with high accuracy.
\end{abstract}

Keywords: Human-structure interaction, free vibration, beam, spring-mass, vibratory characteristics, approximate solution, Galerkin method

\section{Introduction}

It has been experimentally identified that a structure occupied by an individual or a crowd acts at least as a two degree-of-freedom (TDOF) system when the individual or crowd is stationary, such as sitting or standing [1,2]. In other words, the structure can be modeled as a single degree-of-freedom (SDOF) system and the individual or crowd can be considered as another SDOF system [3,4], which forms a TDOF system. Alternatively, an individual or a crowd can be modelled as a concentrated or distributed spring-mass while a structure can be represented as a beam or a plate for theoretical investigation. It is often that a TDOF system is directly used to describe human-structure interaction. Actually, a structure is a continuous system and people distribute on the structure. Thus the link between the actual continuous system and the discrete TDOF system should be established.

It has been noted from practice that only one of the two resonance frequencies of a human-structure was often measured $[1,5,6]$. This is due to the fact that the supporting structure has a light damping ratio while human bodies possess a much larger damping ratio. A recently study on the frequency characteristics of a highly damped TDOF human-structure system [7] shows that the differences between the resonance frequencies and the natural frequency of the TDOF system become larger and the areas where two resonance frequencies exist reduces as the damping ratio of the body increases. However, the damping ratio affects the resonance frequencies rather than the natural frequencies of the system. When human-structure interaction is studied using structural dynamics methods, the equation of motion contains the parameters of the natural frequencies rather resonance frequencies of the system.

\footnotetext{
*Corresponding author. Tel.: +86 25 84316695; Fax: +86 25 85416677; E-mail: dingzhou57@yahoo.com.
} 
Thus it is still necessary and useful to examine the free vibration of an undamped human-structure system and its dynamic characteristics, in particular, a continuous human-structure system.

Some investigators studied the vibratory characteristics of beams attached by concentrated [8-10] or distributed $[11,12]$ rigid masses. Moreover, the free vibration of beams carrying concentrated spring-masses has also been studied [13-16]. It is noted that these models are inapplicable to describe a distributed spring-mass representing a crowd on a structure.

Recently, the authors [17] obtained the exact solution for free vibration of a spring-mass-beam system by modeling a crowd as a distributed spring-mass. It is shown that the beam with distributed spring-mass which has a constant natural frequency can be simulated approximately as a series of TDOF systems where the frequency coupling occurs mainly in the low order of TDOF systems, especially in the first TDOF system if the spring- mass frequency is close to the first beam frequency.

The exact solution [17] provides the benchmark to evaluate the other solutions of coupled vibration of spring-massbeam system. However, it is not easy to apply the exact solution directly to practical applications in engineering due to the requirement of the complicated mathematic knowledge and programming. The present study aims to provide an approximate solution with satisfactory accuracy, which can be easily applied to engineering design and analysis. In this paper, the Galerkin method is used for reaching such a solution. It is well known that the key of using the Galerkin method to obtain a good approximate solution is the selection of the mode shape function. Therefore, static beam functions are derived in which, the effect of both position and magnitude of the mass distribution are considered. An example of hand-worked calculation is provided linking with a comparison to the FE solution. Parametric study is conducted and results are tabled for practical use.

\section{Governing differential equations}

Consider a spring-mass-beam system as shown in Fig. 1. The spring-mass can be distributed or concentrated on the beam. When the spring-mass is distributed on the beam, the differential equations of free vibration of the beam and spring-mass are respectively:

$$
\begin{aligned}
& E I \frac{\partial^{4} y}{\partial x^{4}}+\rho A \frac{\partial^{2} y}{\partial t^{2}}=-\sum_{j=1}^{J} k_{j}\left[U\left(x-x_{1 j}\right)-U\left(x-x_{2 j}\right)\right]\left(y-z_{j}\right) \\
& m_{j} \frac{\partial^{2} z_{j}}{\partial t^{2}}=k_{j}\left(y-z_{j}\right), \quad x_{1 j} \leqslant x \leqslant x_{2 j}
\end{aligned}
$$

where $j$ means the $j$ th section of the distributed mass-springs where the mass and spring are constants and $J$ is the total number of sections of the mass-springs. $y=y(x, t)$ is the displacement of the beam, $E I$ is the flexural stiffness and $\rho A$ is the mass per unit length of the beam. $z_{j}=z_{j}(x, t)$ is the displacement of the distributed spring-mass within the interval $\left[x_{1 j}, x_{2 j}\right], m_{j}$ and $k_{j}$ are the mass and stiffness per unit length of the spring-mass respectively. $U\left(x-x_{1 j}\right)$ and $U\left(x-x_{2 j}\right)$ are the Heaviside functions.

The solutions of the above equations have the following form:

$$
y(x, t)=Y(x) e^{-i \omega t}, \quad z_{j}(x, t)=Z_{j}(x) e^{-i \omega t}
$$

where $\omega$ is the natural frequency of the spring-mass-beam system. $Y(x)$ and $Z_{j}(x)$ are the mode shape functions of the beam and the spring-mass respectively. In the present analysis, it has been assumed that $k_{j} / m_{j}=\omega_{h}^{2}$, i.e. the spring-mass on the beam has a constant natural frequency. This means that the mass and stiffness of the spring-mass system cannot be arbitrary but may vary proportionally in an interval. Substituting Eq. (3) into Eq. (2) gives

$$
Z_{j}(x)=\frac{1}{1-\left(\omega / \omega_{h}\right)^{2}} Y(x), \quad x_{1 j} \leqslant x \leqslant x_{2 j}
$$

Substituting Eqs (3) and (4) into Eq. (1) obtains

$$
E I \frac{d^{4} Y}{d x^{4}}-\rho A \omega^{2} Y=\frac{\omega^{2}}{1-\left(\omega / \omega_{h}\right)^{2}} \sum_{j=1}^{J} m_{j}\left[U\left(x-x_{1 j}\right)-U\left(x-x_{2 j}\right)\right] Y
$$




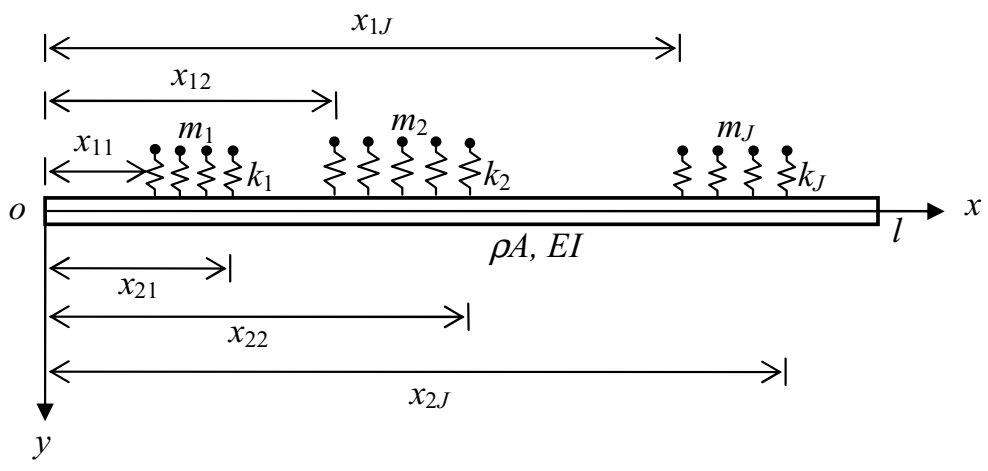

(a) A beam with distributed spring-masses

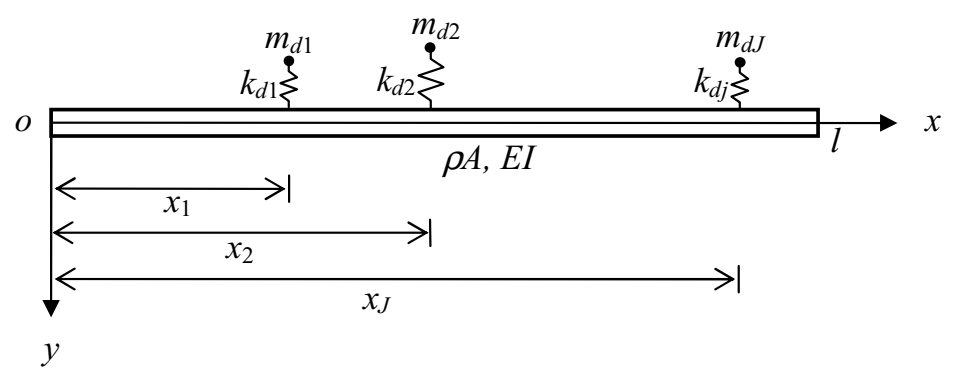

(b) A beam with concentrated spring-masses

Fig. 1. A spring-mass-beam system.

Similarly, when concentrated spring-masses are placed on the beam at $x=x_{j}$, the differential equations of free vibration of the spring-mass-beam system are

$$
E I \frac{d^{4} Y}{d x^{4}}-\rho A \omega^{2} Y=\frac{\omega^{2}}{1-\left(\omega / \omega_{h}\right)^{2}} \sum_{j=1}^{J} m_{d j} \delta\left(x-x_{j}\right) Y
$$

where $\omega_{h}^{2}=k_{d j} / m_{d j}, m_{d j}$ and $k_{d j}$ is the mass and stiffness of the discrete spring-mass respectively. $\delta\left(x-x_{j}\right)$ is the Dirac-delta function.

Equation (5) is the governing differential equation of free vibration of a beam and distributed-spring-mass system and Eq. (6) is that of a beam and concentrated-spring-mass system.

\section{Galerkin solutions}

Multiplying $Y(x)$ to the two sides of Eq. (5) then integrating along the length of the beam, the Galerkin solutions can be obtained as follows

$$
K_{s}-M_{s} \omega^{2}=\frac{\omega^{2}}{1-\left(\omega / \omega_{h}\right)^{2}} M_{h}
$$

where $M_{s}$ and $K_{s}$ are the modal mass and modal stiffness of the beam respectively, $M_{h}$ is the modal mass of the spring-mass. The modal mass and modal stiffness are as follows

$$
M_{s}=\rho A \int_{0}^{l} Y(x)^{2} d x, \quad K_{s}=E I \int_{0}^{l}\left[\frac{d^{2} Y(x)}{d x^{2}}\right]^{2} d x
$$




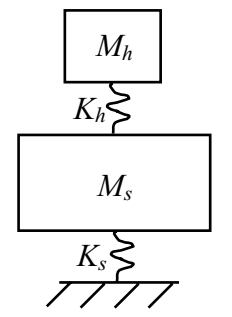

Fig. 2. The approximate TDOF model of a spring-mass-beam system.

The modal mass of the spring-mass is

$$
M_{h}=\sum_{j=1}^{J} m_{j} \int_{x_{1 j}}^{x_{2 j}} Y(x)^{2} d x
$$

From Eq. (7), a pair of natural frequencies of the spring-mass-beam system can be obtained:

$$
\begin{aligned}
& \omega_{1}=\sqrt{\frac{1}{2}\left\{(1+\mu) \omega_{h}^{2}+\omega_{s}^{2}-\sqrt{\left[(1+\mu) \omega_{h}^{2}+\omega_{s}^{2}\right]^{2}-4 \omega_{s}^{2} \omega_{h}^{2}}\right\}}, \\
& \omega_{2}=\sqrt{\frac{1}{2}\left\{(1+\mu) \omega_{h}^{2}+\omega_{s}^{2}+\sqrt{\left[(1+\mu) \omega_{h}^{2}+\omega_{s}^{2}\right]^{2}-4 \omega_{s}^{2} \omega_{h}^{2}}\right\}}
\end{aligned}
$$

where $\mu=M_{h} / M_{s}, \omega_{s}^{2}=K_{s} / M_{s}$. It is demonstrated that a spring-mass-beam system can be approximately expressed by a TDOF system as shown in Fig. 2.

Equation (8) can be written as

$$
M_{s}=a_{s} \rho A l, \quad K_{s}=b_{s} E I / l^{3}
$$

in which,

$$
a_{s}=\int_{0}^{1} Y(\xi)^{2} d \xi, \quad b_{s}=\int_{0}^{1}\left[\frac{d^{2} Y(\xi)}{d \xi^{2}}\right]^{2} d \xi
$$

where $\xi=x / l$. Equation (9) can be written as

$$
M_{h}=\sum_{j=1}^{J} a_{h j} m_{j} l
$$

in which,

$$
a_{h j}=\int_{\xi_{1 j}}^{\xi_{2 j}} Y(\xi)^{2} d \xi
$$

where $\xi_{1 j}=x_{1 j} / l$ and $\xi_{2 j}=x_{2 j} / l(j=1,2, \ldots, J)$.

When spring-masses are concentrated on the beam, Eq. (9) becomes

$$
M_{h}=\sum_{j=1}^{J} m_{d j} Y\left(x_{j}\right)^{2}
$$

Similarly, Eq. (13) becomes

$$
M_{h}=\sum_{j=1}^{J} a_{d j} m_{d j}
$$

where

$$
a_{d j}=Y\left(\xi_{j}\right)^{2}
$$




\section{Basic characteristics of solutions}

The following relationships can be demonstrated [1] from Eq. (11)

$$
\omega_{1} \omega_{2}=\omega_{h} \omega_{s}, \quad \omega_{1}<\left(\omega_{h}, \omega_{s}\right)<\omega_{2}
$$

It can be noted from Eq. (4) that the spring-mass mode is equal to the product of the beam mode and a magnifying factor $1 /\left[1-\left(\omega / \omega_{h}\right)^{2}\right]$. It is known from Eqs (4) and (18) that the vibrations of the spring-mass and the beam are always in the same direction corresponding to $\omega_{1}$ and always in the opposite directions corresponding to $\omega_{2}$. It should be noted that $\omega_{s}={\sqrt{K_{s} / M_{s}}} \neq \omega_{b}$ in the coupled TDOF system, where $\omega_{b}$ is the natural frequency of the bare beam, because $Y(x)$ is the mode shape of the beam coupling with the spring-mass, which is different from that of the bare beam. However, $\omega_{h}$ in the coupled TDOF system is identical as the natural frequency of the independent spring-mass.

The selection of the mode shape function $Y(\xi)$ is fundamental to convert the spring-mass-beam system into a TDOF system with high accuracy. In addition to the boundary conditions, two factors should be considered when determining the mode shape $Y(\xi)$ : the positions and magnitudes of the mass and stiffness of the spring-mass. In the following study, the static beam function will be developed as the approximate mode shape, in which the effect of the mass position and magnitude of the spring-mass on the mode shape are considered. However, the effect of the stiffness of the spring-mass on the mode shape is neglected. The numerical results show later that the effect of the spring stiffness on the mode shape is not significant generally.

\section{Static beam functions}

We consider a static uniform beam under self-weight and several parts of spring-mass in the intervals $\left[x_{1 j}, x_{2 j}\right]$ $(j=1,2, \ldots, J)$. The self-weight of the beam and mass per unit length in the interval $\left[x_{1 j}, x_{2 j}\right]$ are $\rho A g$ and $m_{j} g$ respectively, where $g$ is the gravity acceleration. The static deflection $y(x)$ should satisfy the following governing differential equation:

$$
E I \frac{d^{4} y(x)}{d x^{4}}=g \sum_{j=1}^{J} m_{j}\left[U\left(x-x_{1 j}\right)-U\left(x-x_{2 j}\right)\right]+\rho A g
$$

The general solution of Eq. (19) can be given as:

$$
\begin{aligned}
& y(\xi)=\frac{\rho A g}{E I} Y(\xi) \\
& Y(\xi)=d_{0}+d_{1} \xi+d_{2} \xi^{2}+d_{3} \xi^{3}+\frac{1}{4 !} \sum_{j=1}^{J} \alpha_{j}\left[\left(\xi-\xi_{1 j}\right)^{4} U\left(\xi-\xi_{1 j}\right)-\left(\xi-\xi_{2 j}\right)^{4} U\left(\xi-\xi_{2 j}\right)\right]+\frac{1}{4 !} \xi^{4}
\end{aligned}
$$

where $\alpha_{j}=m_{j} /(\rho A)$. Four unknown coefficients $d_{i}(i=0,1,2,3)$ in the above equation can be uniquely determined using the boundary conditions of the beam, as given in Table 1.

Similarly, we consider a static uniform beam under self-weight and several concentrated spring-masses at $x=x_{j}$ $(j=1,2,3, \ldots, J)$. The weight of each mass at $x=x_{j}$ is $m_{d j} g$. The static deflection $y(x)$ is

$$
Y(\xi)=d_{0}+d_{1} \xi+d_{2} \xi^{2}+d_{3} \xi^{3}+\frac{1}{3 !} \sum_{j=1}^{J} \alpha_{d j}\left(\xi-\xi_{j}\right)^{3} U\left(\xi-\xi_{j}\right)+\frac{1}{4 !} \xi^{4}
$$

where $\alpha_{d j}=m_{d j} /(\rho A l), \xi_{j}=x_{j} / l(j=1,2,3, \ldots, J)$. Four unknown coefficients $d_{i}(i=0,1,2,3)$ in the above equation can also be uniquely determined using the boundary conditions of the beam, as given in Table 2 .

Equations (20) and (21) are called as the static beam functions under the self-weight of the beam and the spring-mass, which will be used as the approximate mode shape to estimate the first coupled frequencies of the spring-mass-beam system and the internal forces (moment and shear force) of the beam. 
Table 1

The coefficients $d_{i}(i=0,1,2,3)$ in Eq. (20) for different boundary conditions (B.C.)

\begin{tabular}{|c|c|c|c|c|}
\hline B.C. & $d_{0}$ & $d_{1}$ & $d_{2}$ & $d_{3}$ \\
\hline S-S & 0 & $\begin{array}{l}\left\{1+\sum_{j=1}^{J} \alpha_{j}\left[\left(1-\xi_{1 j}\right)^{2}(1+\right.\right. \\
\left.2 \xi_{1 j}-\xi_{1 j}^{2}\right)-\left(1-\xi_{2 j}\right)^{2}(1+ \\
\left.\left.\left.2 \xi_{2 j}-\xi_{2 j}^{2}\right)\right]\right\} / 24\end{array}$ & 0 & $\begin{array}{l}-\left\{1+\sum_{j=1}^{J} \alpha_{j}\left[\left(1-\xi_{1 j}\right)^{2}-\right.\right. \\
\left.\left.\left(1-\xi_{2 j}\right)^{2}\right]\right\} / 12\end{array}$ \\
\hline S-C & 0 & $\begin{array}{l}\left\{1+\sum_{j=1}^{J} \alpha_{j}\left[\left(1-\xi_{1 j}\right)^{3}\left(1+3 \xi_{1 j}\right)-\right.\right. \\
\left.\left.\left(1-\xi_{2 j}\right)^{3}\left(1+3 \xi_{2 j}\right)\right]\right\} / 48\end{array}$ & 0 & $\begin{array}{l}-\left\{3+\sum_{j=1}^{J} \alpha_{j}\left[\left(1-\xi_{1 j}\right)^{3}\left(3+\xi_{1 j}\right)-\right.\right. \\
\left.\left.\left(1-\xi_{2 j}\right)^{3}\left(3+\xi_{2 j}\right)\right]\right\} / 48\end{array}$ \\
\hline C-C & 0 & 0 & $\begin{array}{l}\left\{1+\sum_{j=1}^{J} \alpha_{j}[(1-\right. \\
\left.\xi_{1 j}\right)^{3}\left(1+3 \xi_{1 j}\right)-(1- \\
\left.\left.\left.\xi_{2 j}\right)^{3}\left(1+3 \xi_{2 j}\right)\right]\right\} / 24\end{array}$ & $\begin{array}{l}-\left\{1+\sum_{j=1}^{J} \alpha_{j}\left[\left(1-\xi_{1 j}\right)^{3}\left(1+\xi_{1 j}\right)-\right.\right. \\
\left.\left.\left(1-\xi_{2 j}\right)^{3}\left(1+\xi_{2 j}\right)\right]\right\} / 12\end{array}$ \\
\hline C-F & 0 & 0 & $\begin{array}{l}\left\{1-\sum_{j=1}^{J} \alpha_{j}\left[\xi_{1 j}^{2}-\right.\right. \\
\left.\left.\xi_{2 j}^{2}\right]\right\} / 4\end{array}$ & $-\left\{1-\sum_{j=1}^{J} \alpha_{j}\left[\xi_{1 j}-\xi_{2 j}\right]\right\} / 6$ \\
\hline
\end{tabular}

Notes: S means simply supported; C means clamped; F means free.

Table 2

The coefficients $d_{i}(i=0,1,2,3)$ in Eq. (21) for different boundary conditions (B.C.)

\begin{tabular}{|c|c|c|c|c|}
\hline B.C. & $d_{0}$ & $d_{1}$ & $d_{2}$ & $d_{3}$ \\
\hline S-S & 0 & $1 / 24+\sum_{j=1}^{J} \alpha_{d j} \xi_{j}(1-$ & 0 & $-1 / 12-\sum_{j=1}^{J} \alpha_{d j}\left(1-\xi_{j}\right) / 6$ \\
\hline S-C & 0 & $\begin{array}{l}\left.\xi_{j}\right)\left(2-\xi_{j}\right) / 6 \\
1 / 48+ \\
\quad J\end{array}$ & 0 & $-1 / 16-$ \\
\hline C-C & 0 & $\sum_{\substack{j=1 \\
0}} \alpha_{d j} \xi_{j}\left(1-\xi_{j}\right)^{2} / 4$ & $\begin{array}{l}1 / 24+ \\
\sum_{j=1}^{J} \alpha_{d j} \xi_{j}\left(1-\xi_{j}\right)^{2} / 2\end{array}$ & $\begin{array}{l}\sum_{j=1} \alpha_{d j}\left(1-\xi_{j}\right)^{2}\left(2+\xi_{j}\right) / 12 \\
-1 / 12- \\
\sum_{j=1}^{J} \alpha_{d j}\left(1-\xi_{j}\right)^{2}\left(1+2 \xi_{j}\right) / 6\end{array}$ \\
\hline C-F & 0 & 0 & $1 / 4+\sum_{j=1}^{J} \alpha_{d j} \xi_{j} / 2$ & $-\left(1+\sum_{j=1}^{J} \alpha_{d j}\right) / 6$ \\
\hline
\end{tabular}

\section{Determination of factors}

Taking Eq. (20) as the mode shape, the mass factor $a_{s}$ and stiffness factor $b_{s}$ of the beam, and the spring-mass factor $a_{h j}(j=1,2, \ldots, 3, J)$ for the distributed spring-mass can be obtained from Eqs (12) and (14). For the distributed spring-mass, the factors $a_{s}$ and $b_{s}$ in Eq. (12) can be written as without integration:

$$
a_{s}=a_{s 0}+\sum_{j=1}^{J} a_{s 1 j} \alpha_{j}+\sum_{j=1}^{J} a_{s 2 j} \alpha_{j}^{2}, \quad b_{s}=b_{s 0}+\sum_{j=1}^{J} b_{s 1 j} \alpha_{j}+\sum_{j=1}^{J} b_{s 2 j} \alpha_{j}^{2}
$$

The factor $a_{h j}$ in Eq. (14) can be written as:

$$
a_{h j}=a_{h 0 j}+a_{h 1 j} \alpha_{j}+a_{h 2 j} \alpha_{j}^{2}
$$

$a_{s 0}$ and $b_{s 0}$ in Eq. (22) are the mass and stiffness factors of the bare beam, which are independent of the magnitude of the mass and the position of the spring-mass. $a_{h 0 j}$ in Eq. (23) only related to the position of the spring-mass, but not the magnitude of the mass. We can normalize the amplitude of the mode shape with $a_{s 0}=1$. In this case, $b_{s 0}=\lambda_{b}^{2}$ where $\lambda_{b}=\omega_{b} l^{2} \sqrt{\rho A /(E I)}$ is the dimensionless natural frequency of the bare beam. It is seen from Eqs (22) and (23) that if letting $\alpha_{j}=0$, the factors degenerated into those when using the mode shape of bare beam 
Table 3

The mass and stiffness factors of a beam with a uniformly distributed spring-mass

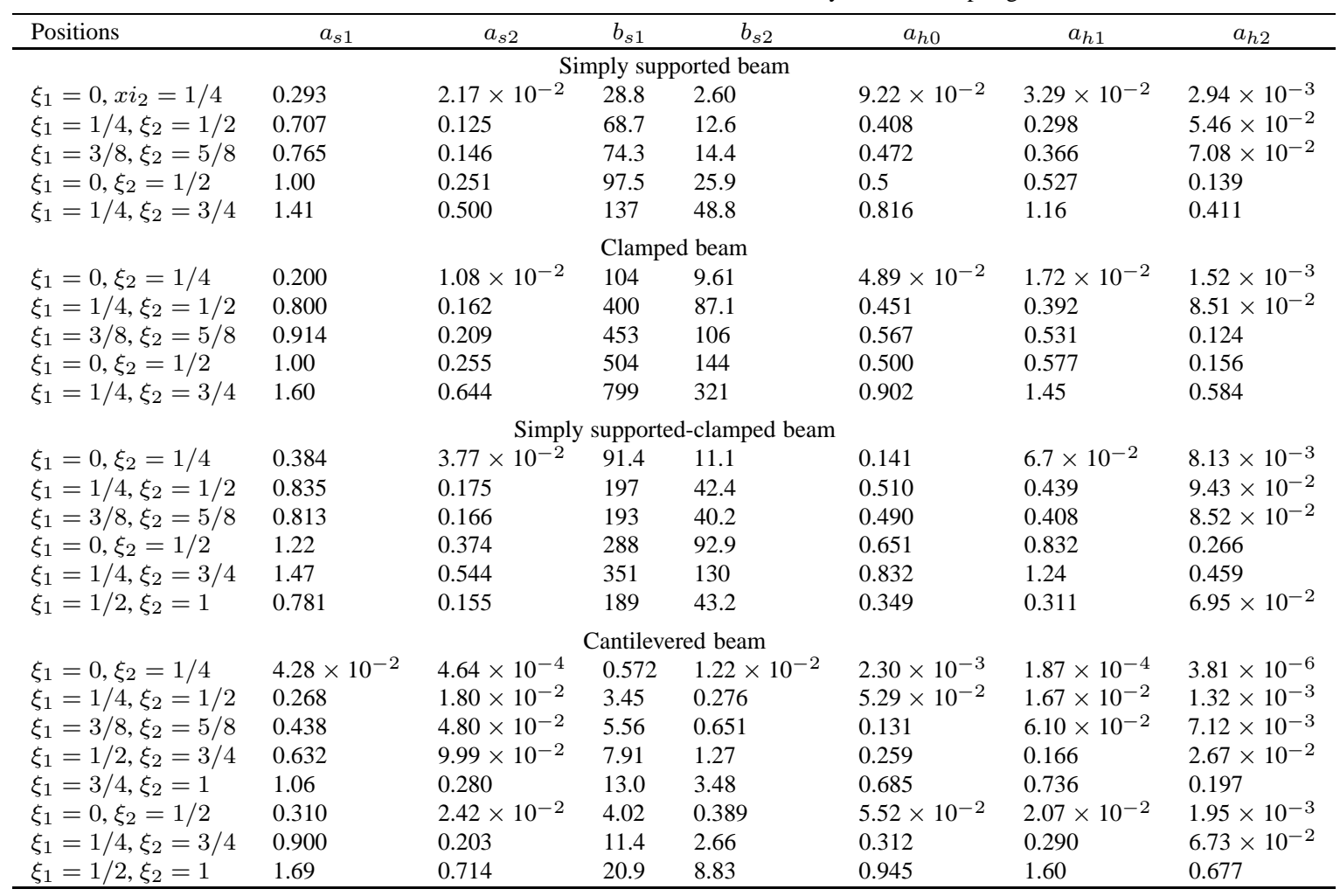

to replace the mode shape of the coupled system. Therefore, the first order and second order terms of $\alpha_{j}$ are the modification to the mode shape of the bare beam.

Similarly, for the concentrated spring-mass, the factors $a_{s}$ and $b_{s}$ in Eq. (12) can be written as:

$$
a_{s}=a_{s 0}+\sum_{j=1}^{J} a_{s 1 j} \alpha_{d j}+\sum_{j=1}^{J} a_{s 2 j} \alpha_{d j}^{2}, \quad b_{s}=b_{s 0}+\sum_{j=1}^{J} b_{s 1 j} \alpha_{d j}+\sum_{j=1}^{J} b_{s 2 j} \alpha_{d j}^{2}
$$

The factor $a_{d j}$ in Eq. (17) can be written as:

$$
a_{d j}=a_{d 0 j}+a_{d 1 j} \alpha_{d j}+a_{d 2 j} \alpha_{d j}^{2}
$$

In the following analysis, the study will focus on the beam with distributed spring-mass in a single region or a single concentrated spring-mass at a point. For simplicity, the subscript to indicate the number of distributed or concentrated spring-mass can be omitted. According to the principle of superposition, a beam with multi-regions of distributed spring-mass or several concentrated spring-masses can be inferred from the typical cases. The mass and stiffness factors of a beam with a distributed spring-mass and a beam with a concentrated spring-mass at different positions on the beam are given in Tables 3 and 4 respectively, where four different boundary conditions are considered. In these calculations, the normalised modes have been used. Using Tables 3 and 4, the calculations can be carried out by handwork.

\section{An example}

To illustrate the use of the method and to verify the method, an example of human-structure interaction is assumed for analysis. Consider a uniform plate of length $5.0 \mathrm{~m}$, width $6.0 \mathrm{~m}$ and thickness $0.3 \mathrm{~m}$. One of the longer sides of the plate is fully fixed and the other sides are free. The material properties of the plate are mass 
Table 4

The mass and stiffness factors of a beam with a concentrated spring-mass

\begin{tabular}{|c|c|c|c|c|c|c|c|}
\hline Positions & $a_{s 1}$ & $a_{s 2}$ & $b_{s 1}$ & $b_{s 2}$ & $a_{d 0}$ & $a_{d 1}$ & $a_{d 2}$ \\
\hline \multicolumn{8}{|c|}{ Simply supported beam } \\
\hline$\xi_{1}=1 / 8$ & 1.20 & 0.367 & 118 & 46.7 & 0.299 & 0.472 & 0.186 \\
\hline$\xi_{1}=1 / 4$ & 2.22 & 1.24 & 217 & 137 & 1.01 & 2.55 & 1.61 \\
\hline$\xi_{1}=3 / 8$ & 2.90 & 2.11 & 282 & 214 & 1.70 & 5.17 & 3.92 \\
\hline$\xi_{1}=1 / 2$ & 3.14 & 2.47 & 305 & 244 & 1.98 & 6.35 & 5.08 \\
\hline \multicolumn{8}{|c|}{ Clamped beam } \\
\hline$\xi_{1}=1 / 8$ & 0.680 & 0.128 & 362 & 158 & $9.02 \times 10^{-2}$ & 0.158 & $6.90 \times 10^{-2}$ \\
\hline$\xi_{1}=1 / 4$ & 2.08 & 1.13 & 1063 & 797 & 0.779 & 2.34 & 1.75 \\
\hline$\xi_{1}=3 / 8$ & 3.33 & 2.81 & 1661 & 1557 & 1.90 & 7.13 & 6.68 \\
\hline$\xi_{1}=1 / 2$ & 3.82 & 3.66 & 1890 & 1890 & 2.46 & 9.84 & 9.84 \\
\hline \multicolumn{8}{|c|}{ Simply supported-clamped beam } \\
\hline$\xi_{1}=1 / 4$ & 2.84 & 2.04 & 671 & 546 & 1.48 & 4.79 & 3.90 \\
\hline$\xi_{1}=1 / 2$ & 3.36 & 2.83 & 796 & 696 & 2.07 & 7.25 & 6.35 \\
\hline$\xi_{1}=3 / 4$ & 1.52 & 0.595 & 373 & 210 & 0.455 & 1.02 & 0.576 \\
\hline \multicolumn{8}{|c|}{ Cantilevered beam } \\
\hline$\xi_{1}=1 / 8$ & 0.133 & $4.46 \times 10^{-3}$ & 1.79 & 0.162 & $3.21 \times 10^{-3}$ & $1.17 \times 10^{-3}$ & $1.06 \times 10^{-4}$ \\
\hline$\xi_{1}=1 / 4$ & 0.498 & $6.25 \times 10^{-2}$ & 6.57 & 1.30 & $4.33 \times 10^{-2}$ & $3.42 \times 10^{-2}$ & $6.76 \times 10^{-3}$ \\
\hline$\xi_{1}=3 / 8$ & 1.05 & 0.276 & 13.6 & 4.38 & 0.184 & 0.238 & $7.70 \times 10^{-2}$ \\
\hline$\xi_{1}=1 / 2$ & 1.74 & 0.754 & 22.1 & 10.4 & 0.488 & 0.919 & 0.433 \\
\hline$\xi_{1}=5 / 8$ & 2.52 & 1.59 & 31.6 & 20.3 & 0.999 & 2.57 & 1.65 \\
\hline$\xi_{1}=3 / 4$ & 3.36 & 2.82 & 41.6 & 35.0 & 1.74 & 5.85 & 4.93 \\
\hline$\xi_{1}=7 / 8$ & 4.23 & 4.47 & 51.9 & 55.7 & 2.70 & 11.6 & 12.4 \\
\hline$\xi_{1}=1$ & 5.11 & 6.53 & 62.3 & 83.1 & 3.89 & 20.8 & 27.7 \\
\hline
\end{tabular}

density $\rho=2400 \mathrm{~kg} / \mathrm{m}^{3}$ and Young's modulus $E=30 \times 10^{9} \mathrm{~N} / \mathrm{m}^{2}$. A crowd of standing people is distributed at a density of four people per square meters equivalent to $300 \mathrm{~kg} / \mathrm{m}^{2}$. The effective body mass contributing to vibration is considered to be two thirds of the body mass [18] with the fundamental natural frequency of $5.5 \mathrm{~Hz}[19]$. Assume that the crowd is uniformly distributed on a half of the plate from its free end (opposite to the fixed) and the distribution does not change along the longer sides of the plate, the analysis of the natural frequencies of the human-structure system can be converted to a spring-mass-beam system when only the fundamental mode of vibration is interested. Thus the equivalent beam has a length $5.0 \mathrm{~m}$, width $6.0 \mathrm{~m}$ and thickness $0.3 \mathrm{~m}$. The distributed spring-mass, representing the crowd, has mass density $m=300 \times 6 \times 2 / 3=1200 \mathrm{~kg} / \mathrm{m}$ and stiffness density $k=m \omega^{2}=1200 \times(2 \pi \times 5.5)^{2}=1.433 \times 10^{6} \mathrm{Nm}^{2}$. The equivalent beam has the properties: $\rho A=2400 \times 6 \times 0.3=4320 \mathrm{~kg} / \mathrm{m}$ and $E I=30 \times 10^{9} \times 6 \times 0.3^{3} / 12=4.05 \times 10^{8} \mathrm{Nm}^{2}$. The corresponding parameters for the simplified calculations are $J=1, \xi_{1}=1 / 2$ and $\xi_{2}=1, \alpha=0.278$. The approximate natural frequency of the bare beam is $\omega_{b} \approx 43.23 / \mathrm{s}$ ( or $f_{b}=6.88 \mathrm{~Hz}$ ) which is obtained using the static beam function.

Substituting the parameters shown in Table 3 into Eq. (22) gives $a_{s} \approx 1.52, b_{s} \approx 18.95$. From Eq. (23), we have $a_{h} \approx 1.44$. Equations (11) and (13) give $M_{s} \approx 3.29 \times 10^{4} \mathrm{~kg}, K_{s} \approx 6.14 \times 10^{7} \mathrm{~N} / \mathrm{m}, M_{h} \approx 8.65 \times 10^{3} \mathrm{~kg}$.

Therefore, we have $\mu \approx 0.263, \omega_{s} \approx 43.18 / \mathrm{s}$ and $\omega_{h} \approx 34.56 / \mathrm{s}$. Substituting these data into Eq. (10) gives $\omega_{1}=30.01 / \mathrm{s}$ and $\omega_{2}=49.72 / \mathrm{s}$, i.e. $f_{1}=4.78 \mathrm{~Hz}$ and $f_{2}=7.91 \mathrm{~Hz}$.

Finite element analysis of the mass-spring-beam system is also conducted using the commercial software package LUSAS [20]. The fundamental frequency of the bare beam is $6.85 \mathrm{~Hz}$ from the FE analysis, which is the same as that from the exact solution. Thin beam elements are used to represent the beam and non-rotational joints are taken to modal the discrete spring-masses. The beam is equally divided into 20 elements and each element has a length of $0.25 \mathrm{~m}$. The distributed spring mass is thus converted into concentrated spring masses with the properties of $m_{c}=1200 \times 0.25=300 \mathrm{~kg}$ and $k_{c}=1.433 \times 0.25=3.58 \times 10^{5} \mathrm{~N} / \mathrm{m}$. The first concentrated spring mass at the centre of the beam and the last at the free end of the beam have a half of the values of the other spring-masses. The corresponding natural frequencies are $4.77 \mathrm{~Hz}$ (the first mode in the $\mathrm{FE}$ analysis) and $7.90 \mathrm{~Hz}$ (the twelfth mode in the FE analysis), which are just the same as the exact solutions. The mode shapes from the second mode to the eleventh mode show only the vibrations of the spring masses. 


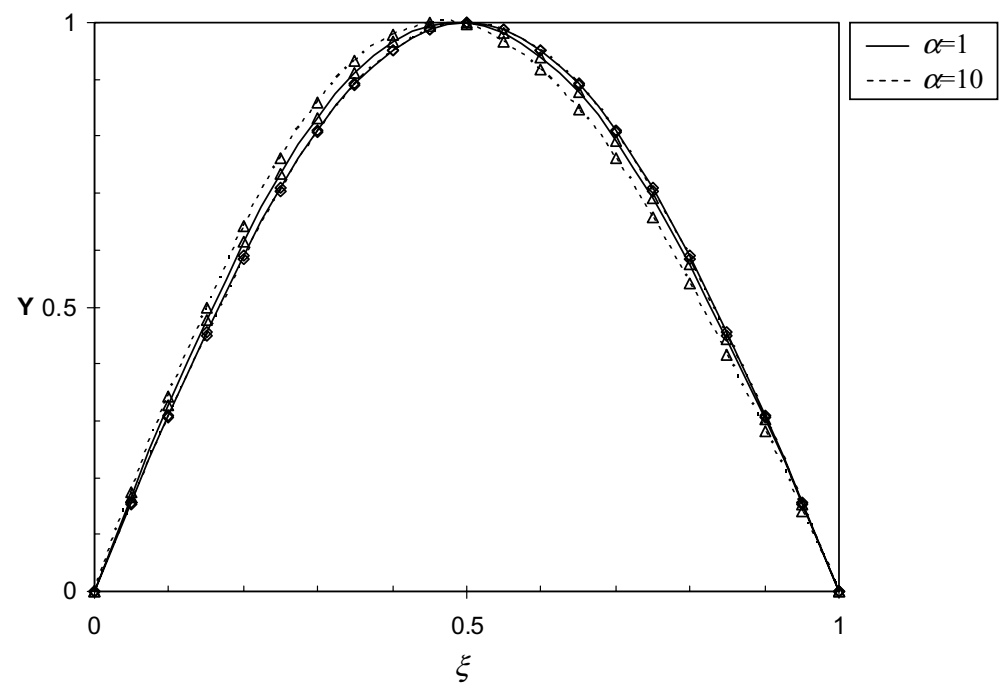

Fig. 3. The mode shapes of a simply supported beam with a uniformly distributed spring-mass in respect to different positions and mass density ratios, $\Delta \longleftarrow\left(\xi_{1}=0.0, \xi_{2}=0.5\right), \diamond \square \square\left(\xi_{1}=0.25, \xi_{2}=0.75\right)$.

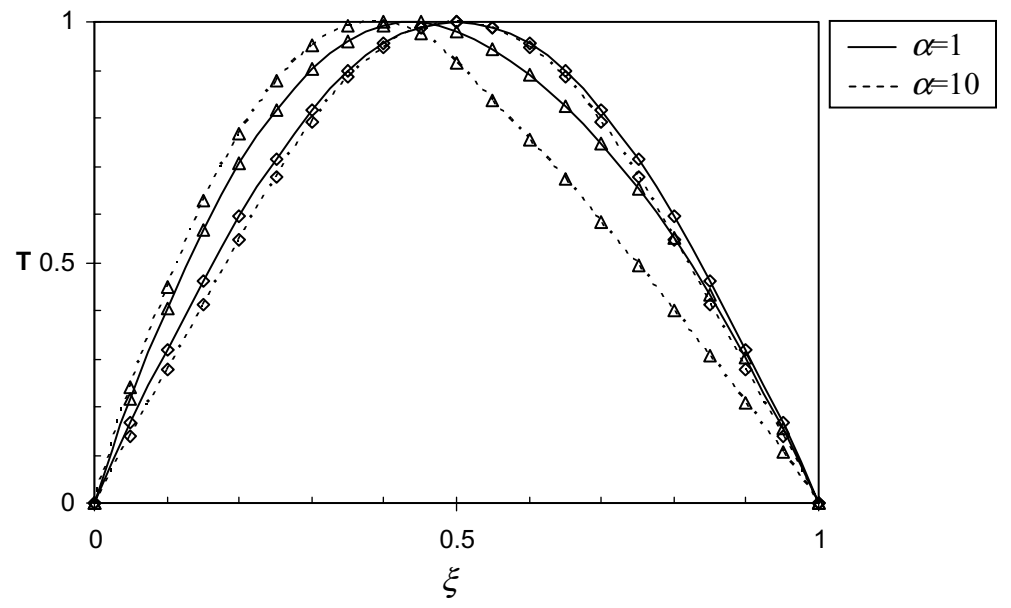

Fig. 4. The mode moments of a simply supported beam with a uniformly distributed spring-mass in respect to different positions and mass density ratios, $\Delta \longleftarrow\left(\xi_{1}=0.0, \xi_{2}=0.5\right), \diamond \square \square\left(\xi_{1}=0.25, \xi_{2}=0.75\right)$.

\section{Characteristics of solutions}

Figure 3 shows the estimated mode shapes $Y(\xi)$ of a simply supported beam with a uniformly distributed springmass on a half length of the beam $\left(\xi_{1}=0.0, \xi_{2}=0.5\right.$ and $\left.\xi_{1}=0.25, \xi_{2}=0.75\right)$ with two different mass ratios $(\alpha=1$ and $\alpha=10$ ). Figures 4 and 5 give the corresponding mode moments $T(\xi)$ and shear forces $V(\xi)$ in the beam respectively, which are approximated by those of the static solutions.

In Eqs (20) and (21), if letting $\alpha_{j}=0$ or $\alpha_{d j}=0$, the mode degenerates into the static beam function of the bare beam. Using this approximate mode, the dimensionless fundamental eigenfrequency $\lambda_{b}=\omega_{b} l^{2} \sqrt{\rho A /(E I)}$ of the beam can be obtained. Table 5 gives the comparison of the dimensionless natural frequencies obtained from the present solution and the exact solution for four kinds of boundary condition. It is seen that the maximum error is less than $0.4 \%$.

It has been demonstrated that when a beam is fully occupied by a uniformly distributed spring-mass, the coupled mode is the same as that of the bare beam [18]. Such a special case can also be recurred using the present solution. 
Table 5

The fundamental eigenfrequency $\lambda_{b}=\omega_{b} l^{2}$

$\sqrt{\rho A /(E I)}$ of bare beams with different bound-

ary conditions

\begin{tabular}{lcccc}
\hline Method & C-C & S-C & S-S & C-F \\
\hline Present & 22.45 & 15.45 & 9.877 & 3.530 \\
Exact & 22.37 & 15.42 & 9.870 & 3.516 \\
Error & $0.36 \%$ & $0.19 \%$ & $0.07 \%$ & $0.40 \%$ \\
\hline
\end{tabular}

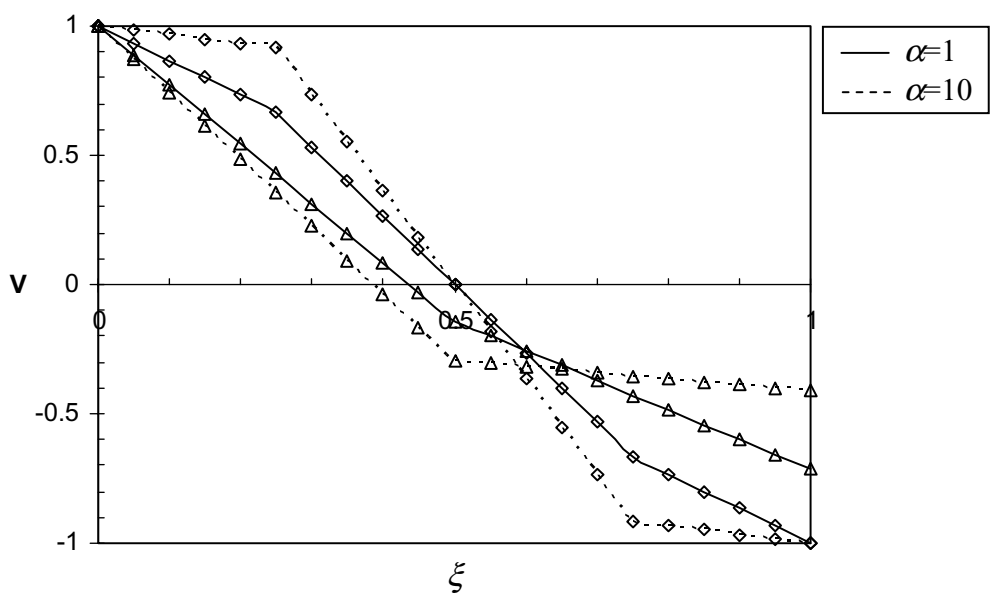

Fig. 5. The mode shear forces of a simply supported beam with a uniformly distributed spring-mass in respect to different positions and mass

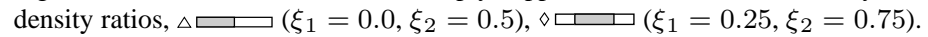

In Eq. (20), if taking $\xi_{1}=0$ and $\xi_{2}=1$ (in such a case, $j=1$ ), the mode automatically degenerates into that of the bare beam, no matter what the mass density is.

Various numerical examples show that in most cases, the accuracy of the first natural frequency is higher than that of the second. It has been numerically demonstrated [17] that the products of a pair of frequencies from the exact solution are close to the product of the natural frequencies of the independent beam and the spring-mass. Therefore, when $\omega_{1}$ has been given by Eq. (10), the second natural frequency $\omega_{2}$ can also be given using the equation

$$
\omega_{2}=\omega_{b} \omega_{h} / \omega_{1}
$$

A number of numerical examples show that $\omega_{2}$ obtained from Eq. (26) is slightly more accurate than that from Eq. (10). This because $\omega_{2}$ from Eq. (10) is generally larger than the exact value. Due to $\omega_{b}<\omega_{s}, \omega_{2}$ given by Eq. (26) is always slightly smaller than that given by Eq. (10), which is generally more close to the exact value.

In order to evaluate the accuracy of the present method, the natural frequencies calculated using the present method and the exact solution are compared. Table 6 compares the dimensionless natural frequencies obtained using the present method and the exact solution. A cantilever with four different positions of the distributed springmass is considered. The mass ratio is selected at $\alpha=m /(\rho A)=1$. Four different stiffnesses are examined, i.e. $\beta=k l^{4} /(E I)=4,9,16$ and 25 . The relation of spring-mass frequency and bare beam frequency is $\omega_{h} / \omega_{b}=\sqrt{\beta / \alpha} \lambda_{b}$. The second frequency parameter $\lambda_{2}$ is obtained from Eq. (26) where the exact value of $\omega_{b}$ from Table 5 is used. It is seen from Table 6 that for the beam with the distributed spring-mass, the present solutions are very close to the exact solutions. The maximum error is less than $1 \%$ for all studied cases. For the beam with the concentrated spring-mass, the first natural frequencies of the present solutions are also close to those of the exact solutions. The maximum error is less than $2 \%$. In most cases, the errors of the second natural frequencies are larger than those of the first natural frequencies. It is shown from Table 6 that in general the error should increase with increasing the stiffness of the spring because the effect of the spring stiffness on static beam function is not considered in the analysis. 
Table 6

The first pair of natural frequencies of a cantilever (C-F) with a uniformly distributed spring-mass in the interval [\&, $\left.\xi_{2}\right], \alpha=m /(\rho A)=1, \beta=k l^{4} /(E I), \lambda=\omega l^{2} \sqrt{\rho A /(E I)}$

\begin{tabular}{|c|c|c|c|c|c|c|c|c|}
\hline \multirow[t]{2}{*}{$\beta, \omega_{h} / \omega_{b}$} & \multicolumn{2}{|c|}{$\xi_{1}=0.0, \xi_{2}=0.5$} & \multicolumn{2}{|c|}{$\xi_{1}=0.25, \xi_{2}=0.75$} & \multicolumn{2}{|c|}{$\xi_{1}=0.5, \xi_{2}=1.0$} & \multicolumn{2}{|c|}{$\xi_{1}=0.0, \xi_{2}=1.0$} \\
\hline & $\lambda_{1}$ & $\lambda_{2}$ & $\lambda_{1}$ & $\lambda_{2}$ & $\lambda_{1}$ & $\lambda_{2}$ & $\lambda_{1}$ & $\lambda_{2}$ \\
\hline $4,0.569$ & 1.974 & 3.562 & 1.873 & 3.755 & 1.692 & 4.157 & 1.681 & 4.183 \\
\hline Exact & 1.973 & 3.558 & 1.873 & 3.747 & 1.691 & 4.159 & 1.679 & 4.189 \\
\hline Error & $0.05 \%$ & $0.11 \%$ & $0.00 \%$ & $0.21 \%$ & $0.06 \%$ & $-0.05 \%$ & $0.12 \%$ & $-0.14 \%$ \\
\hline $9,0.853$ & 2.841 & 3.713 & 2.493 & 4.232 & 2.090 & 5.048 & 2.070 & 5.096 \\
\hline Exact & 2.841 & 3.697 & 2.491 & 4.219 & 2.089 & 5.054 & 2.065 & 5.109 \\
\hline Error & $0.00 \%$ & $0.43 \%$ & $0.08 \%$ & $0.31 \%$ & $0.05 \%$ & $0.08 \%$ & $0.24 \%$ & $-0.25 \%$ \\
\hline $16,1.138$ & 3.277 & 4.292 & 2.789 & 5.042 & 2.273 & 6.186 & 2.250 & 6.251 \\
\hline Exact & 3.268 & 4.271 & 2.784 & 5.013 & 2.269 & 6.195 & 2.242 & 6.272 \\
\hline Error & $0.28 \%$ & $0.49 \%$ & $0.18 \%$ & $0.58 \%$ & $0.18 \%$ & $-0.15 \%$ & $0.36 \%$ & $-0.33 \%$ \\
\hline $25,1.422$ & 3.379 & 5.203 & 2.915 & 6.031 & 2.363 & 7.440 & 2.338 & 7.519 \\
\hline Exact & 3.361 & 5.169 & 2.908 & 5.974 & 2.359 & 7.447 & 2.330 & 7.545 \\
\hline Error & $0.54 \%$ & $0.66 \%$ & $0.24 \%$ & $0.95 \%$ & $0.17 \%$ & $-0.09 \%$ & $0.34 \%$ & $-0.34 \%$ \\
\hline
\end{tabular}

\section{Conclusions}

An approximate estimation is provided in this paper to determine the dynamic characteristics of a beam with distributed or concentrated spring-masses. The static beam function is derived, in which the effect of the magnitude and position of the mass of the spring-mass on the mode shape is considered. By using the static beam function as the approximate mode shape, a pair of coupled frequencies for a spring-mass-beam system is obtained. An example of human-structure interaction is provided to show the use of the proposed method. The conclusions obtained from this study include:

1. The comparison between the present solutions, the exact solutions and FE solutions shows that the present method provides the natural frequencies with satisfactory accuracy.

2. The internal forces of the coupled system are examined using the static beam function. The high accuracy achieved by the function cannot be obtained by using the mode shape of the bare beam. It shows that small variation in mode shapes can lead to large differences on internal forces.

3. The effect of the magnitude and position of the mass of the spring-mass on the mode shape becomes important as the mass ratio increases. The effect should be considered when the coupled frequencies and the internal forces of the spring-mass-beam system are calculated using a TDOF model.

\section{Acknowledgements}

The work reported in this paper has been conducted as part of research project, Human-Structure Interaction Applying body biodynamics into structural dynamics, funded by The Leverhulme Trust, whose support is gratefully acknowledged.

\section{References}

[1] B.R. Ellis and T. Ji, Human-structure interaction in vertical vibrations, Proceedings of the Institution of Civil Engineers-Structures and Buildings 122 (1997), 1-9.

[2] T. Ji, Understanding the interactions between people and structures, The Structural Engineers 81 (2003), 12-13.

[3] T. Ji and B.R. Ellis, Human whole-body models in structural vibration, The 13th ASCE Engineering Mechanics Conference, Baltimore, USA, 13-16 June, 1999.

[4] R. Sachse, A. Pavic and P. Reynolds, Parametric study of modal properties of damped two-degree-of-freedom crowd-structure dynamic systems, Journal of Sound and Vibration 274 (2003), 461-480.

[5] J.M.W. Brownjohn, Energy dissipation from vibrating floor slabs due to human-structure Interaction, Journal of Shock and Vibration 8 (2001), 315-323.

[6] R. Sachse, A. Pavic and P. Reynolds, The influence of a group of humans on modal properties of a structure, 5th European Conference on Dynamics EURODYN, Florence, Italy, 2002. 
[7] D. Wang and T. Ji, Frequency characteristics of a highly damped two degrees-of-freedom system, submitted to the Journal of Sound and Vibration (2006).

[8] R.P. Goel, Vibrations of a beam carrying a concentrated mass, Journal of Applied Mechanics-ASME 40 (1973), 821-822.

[9] K.H. Low, Natural frequencies of a beam-mass system in transverse vibration: Rayleigh estimation versus eigenanalysis solutions, International Journal of Mechanical Sciences 45 (2003), 981-993.

[10] G.B. Chai, K.H. Low and T.M. Lim, Tension effects on the natural frequencies of central-loaded clamped beams, Journal of Sound and Vibration 181 (1995), 727-736.

[11] K.T. Chan, T.P. Leung and W.O. Wong, Free vibrations of simply supported beam partially loaded with distributed mass, Journal of Sound and Vibration 191 (1996), 590-597.

[12] K.T. Chan and J.Z. Zhang, Free vibration of a cantilever tube partially filled with liquid, Journal of Sound and Vibration 182 (1995), $185-190$.

[13] H. Qiao, Q.S. Li and G.Q. Li, Vibratory characteristics of flexural non-uniform Euler-Bernoulli beams carrying an arbitrary number of spring-mass systems, International Journal of Mechanical Sciences 44 (2002), 725-743.

[14] C.A. Rossit and P.A.A. Laura, Free vibrations of a cantilever beam with a spring-mass system attached to the free end, Ocean Engineering 28 (2001), 933-939.

[15] J.J. Wu, Alternative approach for free vibration of beams carrying a number of two-degree of freedom spring-mass systems, Journal of Structural Engineering-ASCE 128 (2002), 1604-1616.

[16] J.S. Wu and H.M. Chou, Free vibration analysis of a cantilever beam carrying any number of elastically mounted point masses with the analytical-and-numerical-combined method, Journal of Sound and Vibration 213 (1998), 317-332.

[17] D. Zhou and T. Ji, Dynamic characteristics of a beam and distributed spring-mass system, International Journal of Solids and Structures 43 (2006), 5555-5569.

[18] T. Ji, A continuous model of the vertical vibration of the human body in a standing position, UK Informal Group Meeting on Human Response to Vibration, Silsoe, UK, 18-20 September, 1995.

[19] L. Wei and M.J. Griffin, Mathematical models for the apparent mass of the seated human body exposed to vertical vibration, Journal of Sound and Vibration 212 (1998), 855-875.

[20] FEA Ltd, LUSAS User Manual, Kingston-upon-Thames, 1993. 

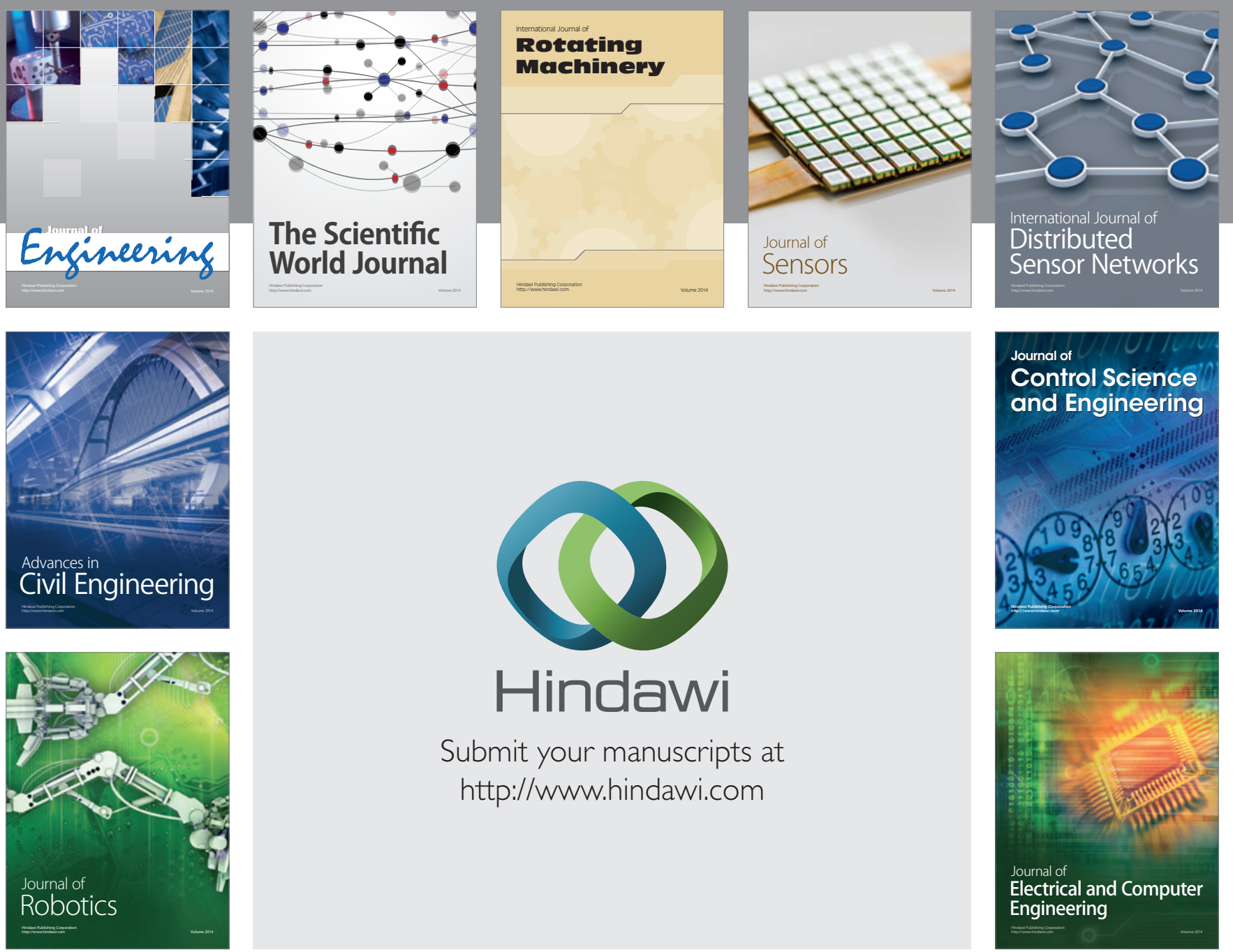

Submit your manuscripts at

http://www.hindawi.com
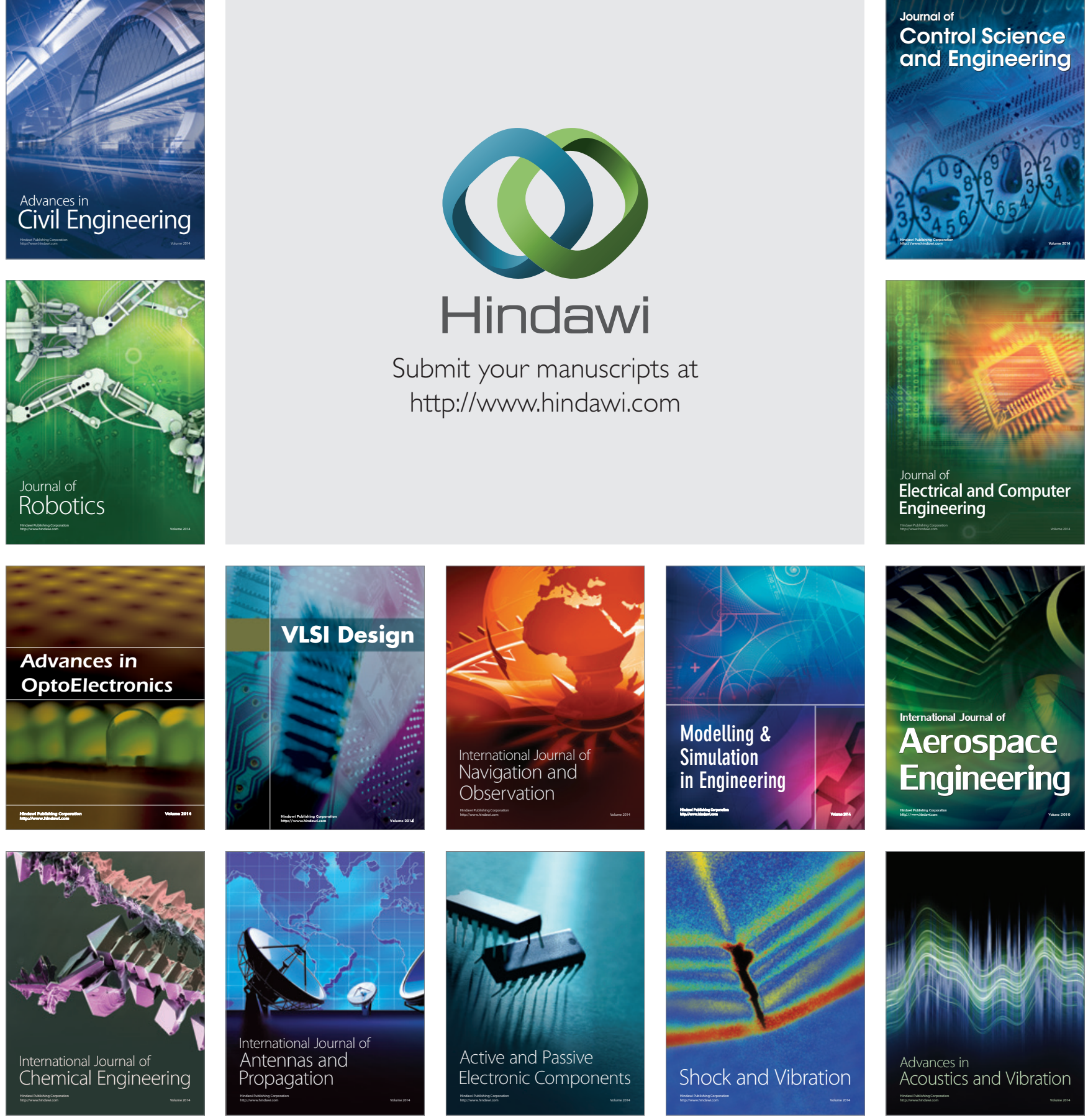